\title{
Theory of Size-Driven Transitions in Displacive and Order-Disorder Ferroelectrics
}

\author{
K. Sheshadrit, Rangan Lahirit Pushan Ayyub**** and Shobo Bhattacharyat \\ Tata Institute of Fundamental Research, Homi Bhabha Road, Mumbai 400005, India
}

(October 18, 1997)

We present a simple theory for structural transitions in displacive ferroelectrics of the perovskite type. In our theory, the competition between the elastic energy cost for the displacement of the homopolar ion from the centrosymmetric position, and the energy gain due to a ferroelectric ordering of the dipoles formed by the ionic displacements, leads naturally to a first-order transition from a paraelectric to a ferroelectric phase. This transition takes place at a certain temperature $T_{c}(L)$ as the temperature is decreased and, at a certain size $L_{c}(T)$ as the size of the system is increased. The transition temperature $T_{c}(L)$ is suppressed as the sample size is reduced, and vanishes for samples below a certain size. For order-disorder ferroelectrics, our theory shows that the suppression of $T_{c}$ by a reduction in system size is not appreciable, a result that is borne out by experiments.

PACS Numbers: 68.35 Rh, 77.84 -s, 77.84 -Dy

\section{INTRODUCTION}

Though the experimental study of finitessize effects in ferroelectric materials has a long history 1 , , the rapid development of advanced synthetic techniques has now made it possible to study different compounds in the form of phase-pure, ultrafine particles with a narrow size distribution. There is also a strong motivation for studying size-limited ferroelectric systems in view of their current and potential applications as sensors, memory elements, nano-robotic and micro-electromechanical devices 3 .

A ferroelectric is termed 'displacive' when the elementary dipoles strictly vanish in the paraelectric phase, and 'order-disorder' when they are non-vanishing but thermally average out to zero in the paraelectric phase. In recent years, the displacive ferroelectric transitions in $\mathrm{PbTiO}_{3}$ and $\mathrm{BaTiO}_{3}$ nanoparticles have been studied in detail. The nature of the size effects observed in both systems is essentially similar. A decrease in the particle size causes monotonic reductions in (a) the transition temperature $T_{c}$, and (b) the tetragonal distortion of the unit cell which characterizes the ferroelectric phase. So, at a low enough particle size, the lattice tends to assume the high temperature, high symmetry, cubic paraelectric structure.

Uchino et al.5 have found that $T_{c}$ (in degrees Centigrade) falls with particle size $L(\mathrm{~nm})$ following the relation

$$
T_{c}(L)=T_{c}(\infty)-\frac{B}{\left(L-L_{c}\right)},
$$

where $T_{c}(\infty)=128(500), \quad B=700$ (588.5), and $L_{c}=$ 110 (12.6) for $\mathrm{BaTiO}_{3}\left(\mathrm{PbTiO}_{3}\right)$.
In a recent study of nanocrystalline $\mathrm{PbTiO}_{3}$ ising dielectric, thermal and structural measurements 6 , it was established that with decreasing particle size: (1) there is a monotonic decrease in the $T_{c},(2)$ the value of the peak dielectric constant $\left(\epsilon_{\max }\right)$ decreases, $(3)$ the ferroelectric transition becomes increasingly diffuse, and (4) the crystallographic unit cell tends towards higher symmetry $(c / a \rightarrow 1)$. Qualitatively similar results have been obtained for $\mathrm{PbZrO}_{3}$, a displacive antiferroelectrid].

It is also instructive to compare finite size effects in displacive and order-disorder ferroelectrics. In electrically insulated samples of sub-micron $\mathrm{KH}_{2} \mathrm{PO}_{4}$, a typical order-disorder system, the depolarization field appeared to prevent thestabilization of ferroelectric ordering below a critical size11. However, a later study of $\mathrm{NaNO}_{2}$ showed clearly that there was no suppression of $T_{c}$ down to $5 \mathrm{~nm}$ in samples suspended in either electrically insulated or conducting media12. It is clear that the size driven effect is much weaker for order-disorder ferroelectrics. This is a feature that our theory also captures (Section V).

In recent years, there have been a few attempts to understand theoretically the nature of size effects in ferroelectrics. Using the phenpmenological LandauDevonshire theory, Zhong et al.13 have shown that the ferroelectric $T_{c}$ should decrease with decreasing size, ultimately leading to a size-driven phase transition from the ferroelectric to the paraelectric phase. Shih et al.14 have considered the effect of incorporating the depolarization energy in the Landau free energy density.

In this paper, we restrict ourselves mainly to the description of quasi-free ferroelectric nanoparticles. The system is assumed to consist of loosely aggregated, unclamped particles which are not electrically isolated. Under such circumstances, we can neglect the effects of external strain and depolarization. This approximates the experimental situation considered by Chattopadhyay et al.5. Specifically, we have selected $\mathrm{PbTiO}_{3}$ as the model system, but the results should apply to other displacivetype systems as well.

$\mathrm{PbTiO}_{3}$ is a classical displacive ferroelectric with a tetragonal perovskite structure $(a=0.3899 \mathrm{~nm}, \quad c=$ $0.4153 \mathrm{~nm}$ ). At room temperature, the $\mathrm{Ti}$ and $\mathrm{O}$ ions are displaced with respect to the $\mathrm{Pb}$ ions, parallel to the polar axis with displacements $d_{\mathrm{Ti}}=.018 \mathrm{~nm}, d_{\mathrm{O}}=.047 \mathrm{~nm} 15$. On increasing the temperature, the tetragonal ferroelectric undergoes a first order transition to a cubic paraelectric phase $(a=c=0.396 \mathrm{~nm})$ at $T_{c}=768 \mathrm{~K}$.

The paper is organised as follows. In section II we present our model and discuss the qualitative physics of the phase transition. Section III gives the details of the 
calculation, and our results are presented in section IV.

In the picture adopted in the present paper, the transition takes place as a result of competition between an ordering tendency of dipoles in adjacent cells and the elastic energy cost associated with the displacement of the atoms. Our main results are the following. We offer a simple physical explanation (see Fig.2) for the firstorder transition in displacive systems driven by temperature and system size. We calculate the strain of the unit cell and make comparison with experiments. While the temperature dependence of the strain (Fig.3) is not in very good agreement with experiments, the size dependence we obtain is in excellent agreement (Fig.5). We show that for order-disorder ferroelectrics, size effects are highly suppressed (section.VD). We present the phase diagram for both kinds of ferroelectrics in the temperature - size plane in Fig.6. The paper ends with a brief discussion of certain features not captured quantitatively in our theory, and prospects for future work (section VI).

\section{THE MODEL AND THE PHYSICAL PICTURE}

A dipole moment is created in each unit cell by the motion of the ionized atom at the centre of the unit cell to an off-centre position. This atom is known as the homopolar atom. In $\mathrm{PbTiO}_{3}$ and $\mathrm{BaTiO}_{3}$ for instance, the Ti atom plays this role. The displacement is accompanied by a distortion of the cubic unit cell to a tetragonal one with sides $a \times a \times c$. The order parameter measured in the experiments $6, \sigma=c / a-1$, obtained from powder $\mathrm{x}$-ray scattering, is a measure of the strain or distortion of the unit cell.

Clearly there is an energy cost at each site associated with the displacement $16 \xi_{i}$ of the homopolar atom. In spite of this cost, the system may find it profitable to undergo a distortion accompanied by an off-center motion of the homopolar ion if there is a negative energy contribution from the interaction energy of dipoles when they are aligned. We describe this energy phenomenologically by an interaction $-J \xi_{i} \xi_{j}$ between nearest neighbours. We refer to this term as the 'Ising' term (see below). For ferroelectrics, $J$ is positive, whereas a negative $J$ will describe antiferroelectrics. In the present work we focus mainly on ferroelectrics (see however section $\mathrm{VI}$ ).

The effective Hamiltonian for the problem is thus the sum of an elastic part and an Ising part. The form of the elastic part may be deduced from simple symmetry considerations. The cost of a displacement $\xi_{i}$ of the homopolar atom at the $i^{\text {th }}$ unit cell and an associated strain $\sigma_{i}(\equiv c / a-1)$ of the unit cell can be written as a powerseries expansion in $\xi_{i}$ and $\sigma_{i}$, at each site. Only even powers of $\xi_{i}$ are allowed in the expansion since the cost cannot depend on which way the atom moves. There is no such restriction for the strain $\sigma$, which is a scalar. We thus arrive at the Hamiltonian

$$
\begin{aligned}
H(\xi, \sigma) & =\sum_{i}\left(\frac{1}{2} k_{2} \xi_{i}^{2}+\frac{1}{4} k_{4} \xi_{i}^{4}+a \sigma_{i}^{2}-b \sigma_{i} \xi_{i}^{2}\right) \\
& -J \sum_{i, j} \xi_{i} \xi_{j} .
\end{aligned}
$$

There is no term proportional to $\sigma_{i}$ alone, since when $\xi_{i}=0$ for all $i$, the system should be in equilibrium with $\sigma_{i}=0$.

An additional consideration has gone into the truncation of the power series. It is expected on symmetry grounds that the thermally averaged value $\langle\sigma\rangle$, of the strain will be an even function of the thermally averaged displacement $\langle\xi\rangle$, and for small distortions, $\langle\sigma\rangle \sim\left\langle\xi^{2}\right\rangle$. This is borne out extremely well from experimental data on $\mathrm{PbTiO}_{3}$ (Fig. 11). From the data we find the empirical relation

$$
\langle\sigma\rangle \simeq A \frac{\left\langle|\xi|^{2}\right\rangle}{a^{2}} .
$$

where $A=3.24$ for $\mathrm{PbTiO}_{3}$. The quadratic relation holds good for other oxides as well17, with different values of $A$.

We may conclude from the above discussion that $\xi^{2}$ and $\sigma$ are of the same order of smallness. The power series expansion in (2.1) thus retains all terms upto $\mathcal{O}\left(\sigma^{2}\right)$ or $\mathcal{O}\left(\xi^{4}\right)$.

Starting with the Hamiltonian (2.1), we can easily integrate out the strain field $\sigma$, to arrive at an effective free energy in terms of the displacements,

$$
\begin{aligned}
F\left(\xi_{i}\right) & =\frac{1}{2} \lambda_{2} \sum_{i} \xi_{i}^{2}+\frac{1}{4} \lambda_{4} \sum_{i} \xi_{i}^{4} \\
& -J \sum_{<i j>} \xi_{i} \xi_{j}
\end{aligned}
$$

where $\lambda_{2}=k_{2}$ and $\lambda_{4}=k_{4}-\frac{b^{2}}{4 a}$.

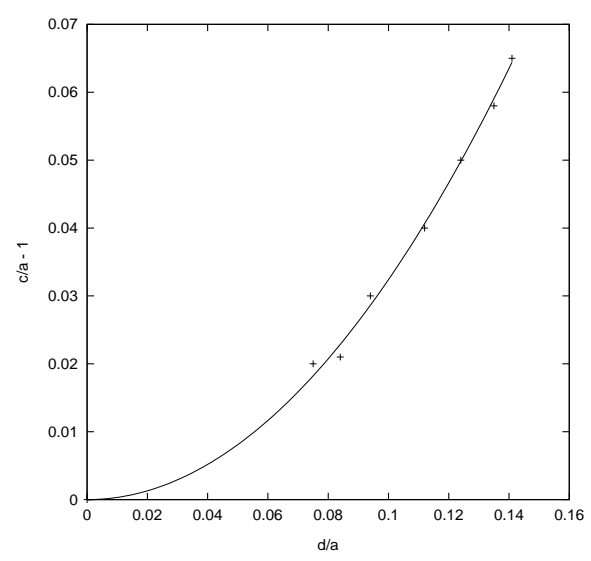

FIG. 1. Experimental values of the strain $\left(\sigma=\frac{c}{a}-1\right)$ plotted as a function of $d / a$, the disptacement $|\xi|$ of the homopolar atom in units of lattice spacing 17 . The points correspond to measurements at different temperatures below $T_{c}$, and the line is the best fit (Eq. 2.2). 


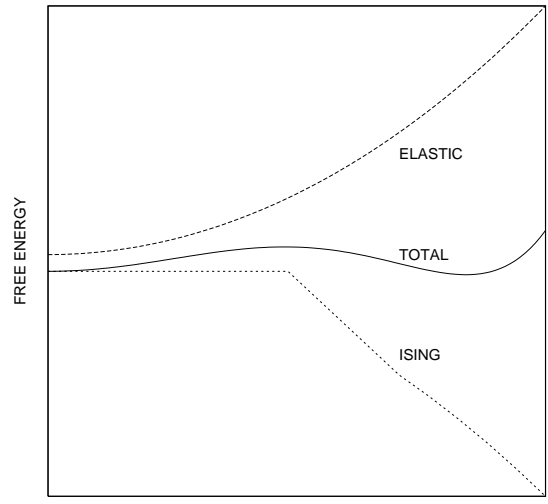

DISPLACEMENT

FIG. 2. Schematic plots of the different contributions to the free energy (2.3). The elastic part (dashed line) has a minimum at the origin. The second minimum is produced by the Ising part (dotted line) which is constant up to a certain displacement and begins to decrease after that. This results in the full free energy with two minima (solid line).

To get a physical picture of the temperature driven transition in bulk samples, we introduce variables $\alpha_{i}$ and $d_{i}$ corresponding to the direction and magnitude of the displacement,

$$
\xi_{i}=d_{i} \alpha_{i}
$$

where $\alpha_{i}(= \pm 1)$ is an Ising variable. With this change of variables, the ferroelectric part of the Hamiltonian (See Eq.(3.1)) is like a compressible Ising term18, since the coupling $\sim J d^{2}$ depends on the displacement. At a temperature $T$, the Ising part cannot be ordered (and hence the system cannot be a ferroelectric) if the coupling is less than $T$. This means that the system can be a ferroelectric only if $d^{2}$ is greater than $\sim T / J$. Whether it actually is a ferroelectric or a paraelectric depends on energetics. This explains why we expect a first-order transition driven by temperature.

To capture this quantitatively, we may perform a partial trace over the $\alpha$ degree of freedom and evaluate a free energy functional of the distortions $d_{i}$ alone, given by

$$
e^{-F(d)}=\operatorname{Tr}_{\alpha_{i}} e^{-H(\alpha, d)}
$$

The global minimum of $F(d)$, determines the thermodynamic phase. We shall see in the following section that this procedure automatically leads to a first order transition.

The size induced transition is also easily understood within this picture. For a system with $N=(L+1)^{3}$ sites, the elastic energy is $\sim N$ and the Ising energy is $\sim N z(L)$ where $z(L)$ is the average coordination number of the cubic lattice of linear size $L$, given by

$$
\begin{aligned}
z(L)= & \frac{1}{(L+1)^{3}}\left[6(L-1)^{3}+30(L-1)^{2}\right. \\
& +48(L-1)+24] .
\end{aligned}
$$

For very large $L, z \rightarrow 6$ and the system, let us say, is ferroelectric provided $T<T_{c}$. If we reduce $L$, this reduces $z(L)$, and the magnitude of the Ising part decreases as a result. This makes the ferroelectric phase unstable at small size and the system becomes a paraelectric.

In particular, at zero temperature, when there are no thermal fluctuations, $\alpha_{i}$ is the same at all sites, and the free energy is simply

$$
f(d)=\frac{1}{2}\left(\lambda_{2}-J z(L)\right) d^{2}+\frac{1}{4} \lambda_{4} d^{4},
$$

which describes a second order transition driven by size when

$$
J z\left(L_{c}\right)=\lambda_{2}
$$

at a certain critical size $L_{c}$.

\section{CALCULATIONS}

In this section we present a mean-field theory for our model. The effective Hamiltonian (2.3) expressed in terms of $d_{i}$ and $\alpha_{i}$ is

$$
\begin{aligned}
F(d, \alpha) & =\frac{1}{2} \lambda_{2} \sum_{i} d_{i}^{2}+\frac{1}{4} \lambda_{4} \sum_{i} d_{i}^{4} \\
& -J \sum_{<i j>} d_{i} d_{j} \alpha_{i} \alpha_{j} .
\end{aligned}
$$

Recall that $d_{i}$ is the magnitude of the displacement of the homopolar ion in the $i^{\text {th }}$ unit cell and $\alpha_{i}$ is its sign. We now perform a trace over the $\alpha$ variables at the meanfield level, to get

$$
\begin{aligned}
F\left(d_{i}\right) & =\frac{1}{2} \lambda_{2} \sum_{i} d_{i}^{2}+\frac{1}{4} \lambda_{4} \sum_{i} d_{i}^{4} \\
& +\frac{1}{2} J \sum_{<i j>} m_{i} m_{j} d_{i} d_{j} \\
& -T \sum_{i} \ln 2 \cosh \left(\frac{J d_{i} \sum_{j} m_{j} d_{j}}{T}\right) .
\end{aligned}
$$

The thermal average $m_{i}=\left\langle\alpha_{i}\right\rangle$ is determined by the self consistency equation

$$
m_{i}=\tanh \left(\frac{J d_{i} \sum_{j} m_{j} d_{j}}{T}\right)
$$

In this paper, we resort to the approximation $d_{i}=d$, i.e., we assume that the homopolar atom displaces by the same amount in all the unit cells. Therefore, the free energy per unit cell $f\left(d_{i}\right)=F\left(d_{i}\right) / N$ is given by (see Eq. (3.2)

$$
f(d)=e(d)+I(d),
$$

where we have separated the elastic part 


$$
e(d)=\frac{1}{2} \lambda_{2} d^{2}+\frac{1}{4} \lambda_{4} d^{4},
$$

and the Ising part

$I(d)=J d^{2} \frac{1}{N} \sum_{<i j>} m_{i} m_{j}-\frac{1}{N} \sum_{i} T \ln 2 \cosh \left(\frac{J d^{2} \sum_{j} m_{j}}{T}\right)$.

For a bulk system $(L \rightarrow \infty)$, the Eq.(3.3) gives a uniform solution $m_{i}=m$. We thus have

$$
I(d)=\frac{1}{2} J z m^{2} d^{2}-T \ln 2 \cosh \left(\frac{J z m d^{2}}{T}\right) .
$$

where $z(=6)$ is the coordination number of the cubic lattice.

The schematic plots of $e(d), I(d)$ and $f(d)$ are shown in Fig. 2. It can be seen that the elastic part is an increasing function of the displacement with a minimum at $d=0$, whereas the Ising part is constant, equal to $-T \ln 2$, for $d<d_{c}=\sqrt{T / J z}$ and decreases (i.e. increases in magnitude, being negative always) for larger $d$. Indeed, for $d<d_{c}, \quad m=0$ (see Eq. (3.3)) and therefore $I(d)=-T \ln 2$. As a result of this behaviour of $I(d)$, the free energy develops a second minimum at some $d=d_{0}$, which is found by solving the equation $\partial f / \partial d=0$. For large $d, \quad I(d) \sim-d^{2}$, so that the quartic term in $e(d)$ dominates for large $d$, resulting in a stable free energy function as shown in Fig. 2. It is this two-minima structure of the free energy that gives rise to a first-order transition between the ferroelectric (the $d=d_{0}$ minimum) and paraelectric (the $d=0$ minimum) phases.

Minimising the free-energy in Eq.(3.4) with respect to $d$, we get

$$
\lambda_{2} d+\lambda_{4} d^{3}-J z m^{2} d=0
$$

corresponding to the two minima, one at $d=0$ and the other at

$$
d_{0}=\sqrt{\frac{J z m^{2}-\lambda_{2}}{\lambda_{4}}} .
$$

At the first-order transition, the two minima coexist, i.e., $f(0)=f\left(d_{0}\right)$, or

$$
\frac{1}{2} \lambda_{2} d_{0}^{2}+\frac{1}{4} \lambda_{4} d_{0}^{4}+\frac{1}{2} J z m^{2} d_{0}^{2}-T_{c} \ln \cosh \left(\frac{J z m^{2} d_{0}^{2}}{T_{c}}\right)=0 .
$$

We need three conditions to fix the three parameters of our theory $\lambda_{2}, \lambda_{4}$, and $J$. The last two equations 3.9 and 3.10$)$ supply two of these conditions; in these we use the experimental values of $T_{c}$ and $d_{0}$. The third condition is the equation $\lambda_{2}=J z\left(L_{c}\right)$ derived at the end of the last section.
From experiments on $\mathrm{PbTiO}_{3}$, we have $d_{0}=0.299 \AA$, $T_{c} \simeq 768 \mathrm{~K}$, and from the work of Zhong et al.13, $L_{c} \simeq$ 10. Using these in the three equations above, we obtain $J=4.345909 \times 10^{4} \mathrm{~K}^{-2}, \lambda_{2}=2.3718 \times 10^{5} \mathrm{~K}^{-2}$, and $\lambda_{4}=2.6529 \times 10^{5} \mathrm{~K}^{-4}$.

Now it is not surprising that since $J z d_{0}^{2} / T_{c} \simeq 30.27$, we get $m \simeq 1$ for all the sites (see Eq.(3.3)). This in fact turns out to be the case also for a finite lattice, so that the equations (3.9) and (3.10) can be used even in this case by replacing $z$ by $z(L)$. The results from this completely agree with the results of solving Eq.(3.3) explicitly for a finite lattice with open boundary conditions, which we have performed numerically.

To obtain the phase diagram, we calculate $d_{0}(T)$ for each $L$ from equations (3.9) and (3.10) (with $z(L)$ replacing $z$ ).

\section{RESULTS}

Fig.3 shows our result for the strain as a function of temperature for the bulk case (solid line); the experimental data 17 are also shown (crosses). The lack of quantitative agreement is ascribed to the fact that in our meanfield theory, the ferroelectric is completely saturated, i.e., $m \simeq 1$ at all $T<T_{c}$.

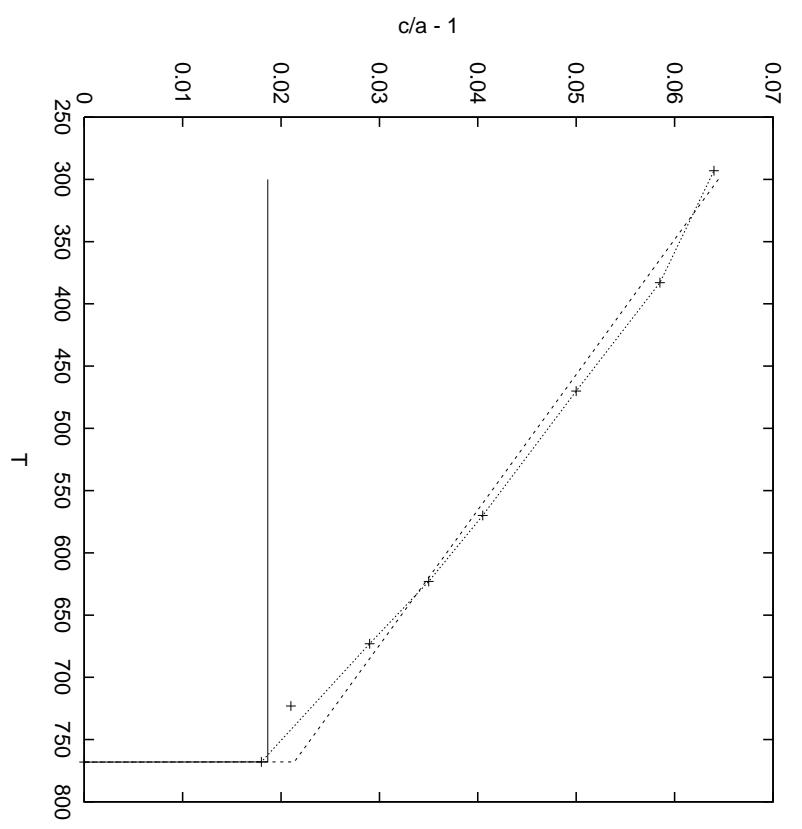


FIG. 3. The strain plotted as a function of temperature $(T)$ for bulk samples. The crosses are the experimental points and the solid line is from the theory. The dotted line is obtained by assuming a weak linear temperature dependence for $\lambda_{2}$, and the dashed line is by assuming a temperature dependent $\lambda_{4}$ (Fig.4).

We can make up for this disagreement in two ways. One possibility is to assume a weak temperature dependence for $\lambda_{2}$, of the form $A T+B$; For $B=5.45 J, \quad A=$ $5.5 \times 10^{-4} J, \quad \lambda_{4}=1.23 J \AA^{-2}$ and $J=1.65 \times 10^{5} \mathrm{~K}^{\circ}{ }^{-2}$ ( $T$ measured in degrees Kelvin) this leads to the dotted line shown in Fig.3. We find that while this makes the order parameter temperature dependent, it also makes it $J$-independent and therefore size-independent, though the size-driven transition still remains.

Alternatively, we can assume a temperature dependence for $\lambda_{4}$ which can be calculated by requiring that the order parameter agree with experiment. This turns out to be a rather strong temperature dependence for $\lambda_{4}$ which we show in Fig. A. Our subsequent results are obtained by assuming that the parameters $\lambda_{2}$ and $\lambda_{4}$ are independent of temperature.

We have shown in Fig. 5 a plot of strain calculated as a function of system size at a temperature of $300 \mathrm{~K}$, along with the corresponding experimental data 6 . In this graph, we have normalized the calculated strain in a way so as to match the experimental value for the bulk system at this temperature. We obtain excellent agreement with experiment for the size dependence of the strain.

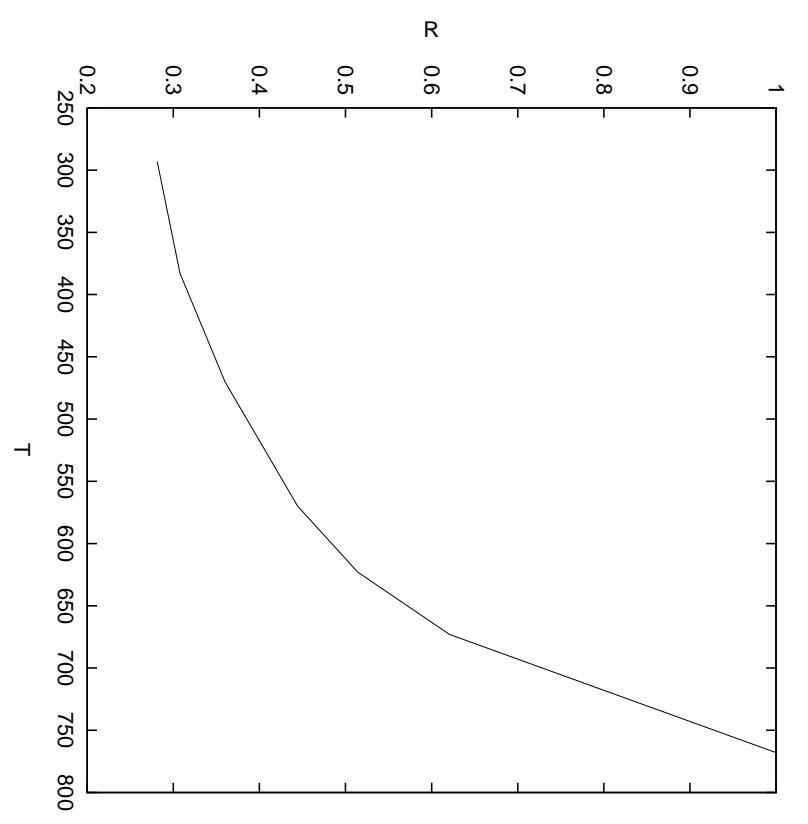

FIG. 4. The ratio $R=\lambda_{4}(T) / \lambda_{4}\left(T_{c}\right)$ plotted as a function of temperature. This dependence leads to the dashed line shown in Fig. 3 . 


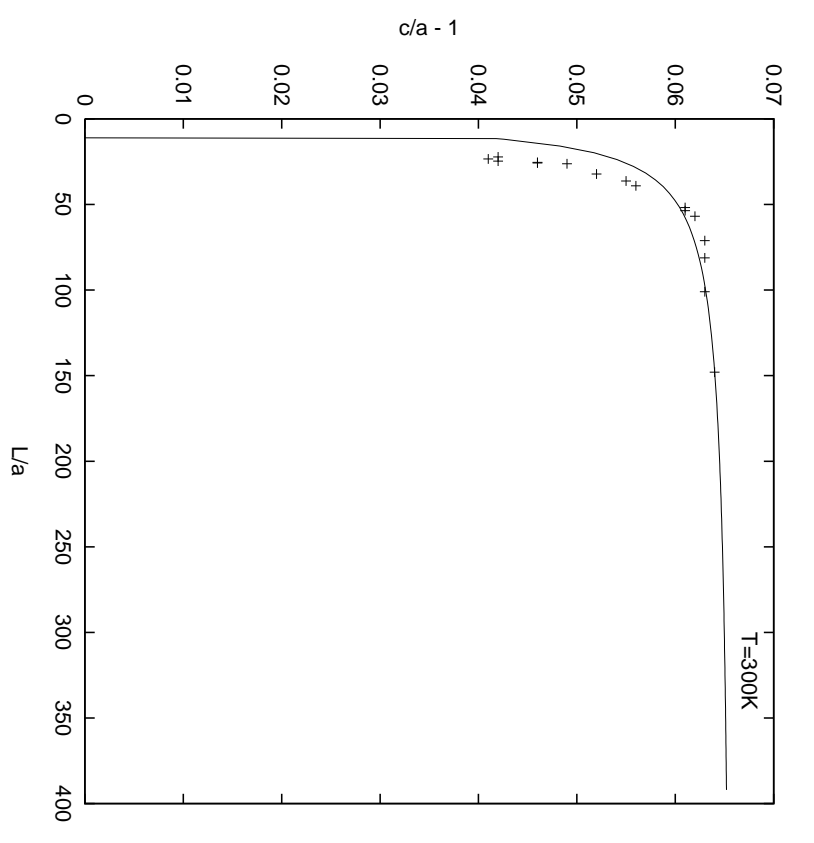

FIG. 5. The strain in the tetragonal phase $\left(\frac{c}{a}-1\right)$ as a function of reduced system size at room temperature. The solid line shows the strain calculated frop our theory (see text). The crosses are experimental points 0 .

Fig.6 shows our phase diagram in the temperature size plane, obtained by calculating strain. The phase boundary for displacive systems (solid line) is a line of first-order transitions for all $T>0$, and the size-driven transition at $T=0$ is continuous, as noted above. It can be seen from the phase diagram that our theory predicts a suppression of $T_{c}$ as the system size is reduced, in agreement with experiments on displacive systems and also other theoretical studies on these systems.

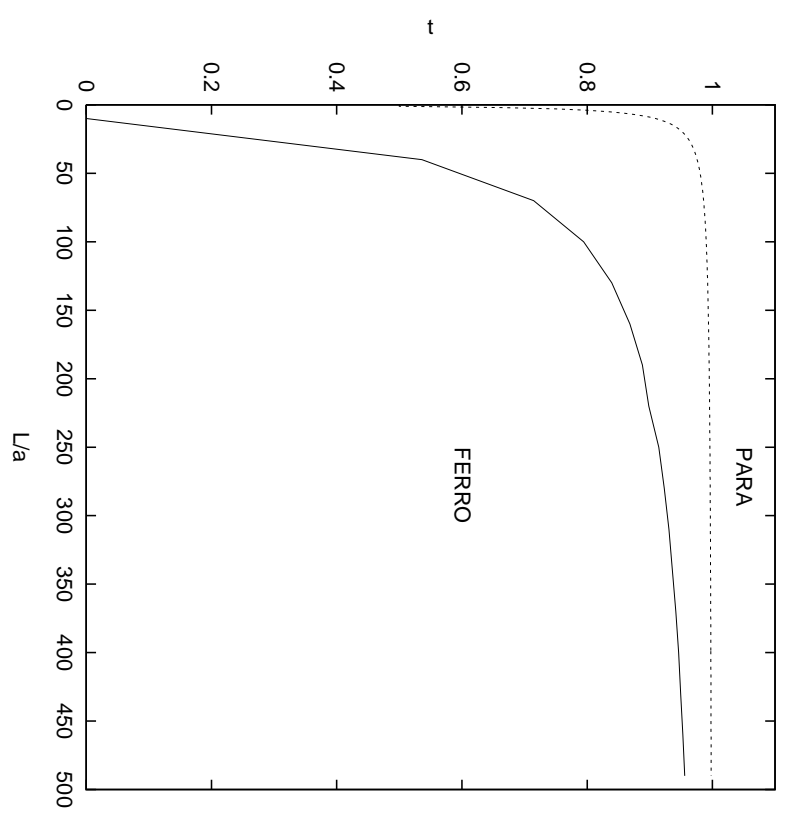

FIG. 6. The phase diagram of ferroelectric oxides in the temperature-size plane obtained within our theory. We have plotted the reduced temperature $t=T_{c}(L) / T_{c}(\infty)$ against the reduced particle size. The solid line is for displacive systems and is a first-order line separating the tetragonal ferroelectric phase from the cubic paraelectric phase. It can be seen that there is an appreciable suppression of $T_{c}$ as the system size is reduced, and $T_{c}$ is zero below a certain size. The dotted line is for order-disorder systems and is a line of second-order transitions separating the low-temperature ferroelectric phase from the high temperature paraelectric phase. The transition temperature $T_{c}$ remains practically equal to its bulk value down to very small system sizes, and the size effects are highly suppressed in this case. In particular, the system remains in the ordered phase below $T_{c} / 2$ for all sizes.

\section{ORDER-DISORDER FERROELECTRICS}

For order-disorder ferroelectrics, the local potential experienced by the homopolar atom has a double well structure 20 , i.e., there is a local maximum at $d=0$ and two minima at $d= \pm d_{0}$. This corresponds to $\lambda_{2}<0$ within our approach.

In this case, it is easy to see that size effects are highly suppressed. Firstly, we note that the Ising term can never lead to a minimum at $d=0$ at any finite temperature. Therefore an increase in temperature can destroy the ferroelectric order via a continuous transition. The distortion continues to have the value given in Eq.(3.9), but 
$m=\langle\alpha\rangle$ vanishes when $J z(L) d_{0}^{2} / T=1$. The last condition can be met either by changing $T$ or by changing system size. For the bulk system $(\mathrm{z}=6)$, this is satisfied for $T=T_{c}$. We can therefore write $z(L) / 6 \simeq T / T_{c}$, where $L$ is the size at which there is a size-driven transition at temperature $T$. Note that when expressed in terms of the reduced transition temperature $t=T_{c}(L) / T_{c}(\infty)$ the above equation (which describes the boundary between the ferroelectric and paraelectric phases) is independent of our model parameters. If $T / T_{c}$ is about 0.9 (say), then we have to go to such small systems as $L \sim 10$ (see Eq.(2.6) to see size effects.

Typically, the lattice spacings in these systems are $~$ $4 \AA$, so we have to get samples of size $\sim 4 \mathrm{~nm}$, to have observable size effects, even at so high a temperature as $0.9 T_{c}$. At lower temperatures the critical sizes are even smaller. In particular, for $T<0.5 T_{c}$, we do not expect any size-driven transition, since $z(L)$ cannot be less than 3. The experiment on $\mathrm{NaNO}_{2} 12$ that we referred to in Sec. If reports that $T_{c}$ is not suppressed down to sizes of 5 $\mathrm{nm}$.

We have shown the phase diagram for order-disorder ferroelectrics also in Fig.6, and the difference between the two kinds of ferroelectrics is very instructive. We attribute the suppression of size effects in the case of orderdisorder ferroelectrics to the absence of a structural transition accompanying the ferroelectric transition. Indeed, at any temperature, one has to go to much smaller samples to meet the requirement $J z(L) d_{0}^{2} / T=1$ (which is the transition condition for order-disorder systems) than the co-existence condition Eq.(3.10) (which is the transition condition for displacive systems).

\section{DISCUSSIONS}

Although our model gives a useful physical picture of both temperature and size driven transitions, it is rather too simple to provide perfect quantitative agreement. We have made several simplifying assumptions, the most significant being that the displacement has the same magnitude throughout the sample. This is not necessarily true, especially for small systems where the displacement at the surface could be different from that it is in the bulk. This, and the subsequent neglect of thermal fluctuations, turn out to be rather drastic approximations. The strongly first-order character of the transition (i.e. the very weak, almost nonexistent temperature dependence of the order parameter) is probably an artifact of our mean-field approximation.

An interesting question, which cannot be answered within the framework of our theory, is whether the transition remains first-order beyond mean-field theory. Further, since our theory is an effective single-site theory, it cannot capture capture the phenomenon of mode softening that is observed in these system 22 .

We do not have the effect of depolarization field in our theory. The depolarization field, under certain circumstances, caplead to better quantitative agreement with experiment 14 . However, in the experimental situation that we are describing 9 , the effect of the depolarization field should be negligible. While we have not made explicit the role of long range interactions between dipoles, this will simply lead to a reparametrisation of $J$ within the simple version of our theory presented in this paper.

The mean-field theory that we have attempted on the Ising part of the Hamiltonian consists in reducing the lattice problem to an effective single-site problem. This led to a very weakly temperature order parameter as we have seen. We have also performed calculations based on the Bethe-Peierls approximation 23 , which takes into account short range correlations between dipoles. However, this did not lead to any hetter temperature dependence for the order parameter 24 .

There are certain qualitative merits of our theory that are worth emphasizing. For example, our theory can be easily extended to include antiferroelectric oxides, simply by having a negative $J$. The ordered phase will have a nonzero value for an antiferroelectric order parameter such as sublattice magnetization $\alpha_{\mathrm{S}}$. The molecular field at any site $i$, due to the nearest neighbours, will be opposite in sign to the order parameter at that site, so that the product of the molecular field and $J$ will have the same sign as for the ferroelectric case. The theory as worked out above in terms of displacements will go through without any further change, and the results will be identical to that for ferroelectrics, with $J$ replaced by $|J|$. Recent experimental studies of size-driven transitions in antiferroelectric materials report results very similar to those as ferroelectrics, confirming our expectation.

We can explain the form of Eq.(1.1) for the size-driven transitions quite simply in our theory. For the size-driven transition, we get from Eq.(3.10), upon replacing $\lambda_{2}, \lambda_{4}$, and $J$ by their values determined from bulk data, an equation of the form $\ln \cosh \left(z(L) / T_{c}\right)=z(L) / T_{c}-A$, for some constant $A$. It is clear that this equation will have a solution of the form (1.1) for large $L$ since $z(L)$ is given by Eq.(2.6). The actual numbers appearing in Eq.(1.1) will of course depend on the system we address.

Our theory has three fitting parameters, whereas the phenomenological Landau theories 13.14 have about twice as many. This circumstance is clearly because our theory is based on a microscopic model which identifies the different contributions to the system free energy as being due to the elastic and ferroelectric parts. As a result, we are also able to describe the structural transition in the displacive systems in addition to the ferroelectric transition, to which it is related. The chief qualitative merit of our theory is that the first-order transition appears in a very natural way, with minimal assumptions about the form of the elastic part of the Hamiltonian. With the lowest order nonlinear elastic term (i.e. fourth order), we are able to describe the first-order transition. This is an essential difference with earlier approaches based on a Landau expansion of the free energy. 
Finally, we have shown that our theory can describe size-driven transitions in order-disorder systems as well as displacive ones. Our simple estimate for the critical size at which size effects become important in these systems is in excellent agreement with data on $\mathrm{NaNO}_{2} 12$. Our approach is superior to the Landau theory for sizedriven transitions 13 since size effects in order-disorder systems can be addressed in our framework.

In conclusion, we have presented a simple unified picture for structural transitions in displacive and orderdisorder ferroelectric oxides, which captures the physics of both temperature-induced transitions in the bulk and size induced transitions in nanoparticles. The size effects are understood in a simple way as being the result of smaller coordination number near the surface of the particle. Our mean-field approximation gives qualitatively good results, except for the temperature dependence of the order parameter. Future work will center around incorporating the effect of thermal fluctuations and getting closer agreement with experiments.

\section{ACKNOWLEDGMENTS}

We would like to thank Mustansir Barma whose suggestions and critical remarks have gone a long way in helping us understand the intricacies of the problem.We thank Deepak Dhar for being the perfect bouncing board. We have also benefited from discussions with Soma Chattopadhyay, Chandan Dasgupta, Rahul Pandit, T.V. Ramakrishnan, Mohit Randeria, Madan Rao and Nandini Trivedi. We thank Mustansir Barma, Deepak Dhar, and Arun Paramekanti for their comments on the manuscript. SB acknowledges hospitality of TIFR.

* Theoretical Physics Group; email: shesh@theory.tifr.res.in

$\dagger$ Theoretical Physics Group; email: rangan@theory.tifr.res.in

** Materials Research Group; email: pushan@tifrvax.tifr.res.in

¥ Permanents address: NEC Research Institute, 4, Independence Way, Princeton, New Jersey 08540, USA; email: shobo@research.nj.nec.com

${ }^{1}$ M Anliker ,H.R. Brugger and W. Känzig, Helv. Phys. Acta 27, 99 (1954).

${ }^{2}$ W. Känzig , Phys. Rev. 98, 549 (1955).

${ }^{3}$ M.H. Francombe in Physics of Thin Films: Mechanic and Dielectric Properties, edited by M.H. Francombe and J.L. Vossen (San Diego: Academic, 1993) pp 225-300.

${ }^{4}$ M.E. Lines and A.M. Glass, Principles and Applications of Ferroelectrics and Related Materials (Clarenden Press, Oxford 1977).
${ }^{5}$ K. Uchino, E. Sadanaga and T. Hirose 1989 J. Amer. Ceram. Soc. 721555

${ }^{6}$ S. Chattopadhyay, P. Ayyub, V.R. Palkar and M. Multani Phys. Rev. B 52, 13177 (1995).

${ }^{7}$ S. Chattopadhyay, P. Ayyub, V. R. Palkar, A. V. Gurjar, R. M. Wankar, and M. S. Multani, J. Phys. Condensed Matter 9, 8135 (1997).

${ }^{8}$ K. Ishikawa, K. Yoshikawa, and N. Okada, Phys. Rev.B 37, 5852 (1988). The trends in the experiments of Chattopadhay et al $\mathbf{6}$ agree with those in the earlier expriments described above. In this paper, for concrteness, we shall refer to the experimental results of Chattopadhayay et al..

${ }^{9}$ G. Burns and B.A. Scott, Phys. Rev. Lett.25 167 (1970); Phys. Rev.B 7, 3088 (1973).

${ }^{10}$ W.L. Zhong, B. Jiang, P.L. Zhang, J.M. Ma, H.M. Cheng, Z.H. Yang and L.X. Li, J. Phys.: Condens. Matter 5, 2619 (1993). R.M. Wankar, and M. Multani, J. Phys.: Condens. Matter (in press).

${ }^{11}$ C. Jacard, W. Känzig and M. Peter, Helv Phys Acta 26, 521 (1953).

${ }^{12}$ P. Marquardt and H. Gleiter, Phys. Rev. Lett. 48, 1423 (1982).

13 W.L. Zhong, Y.B. Wang, P.L. Zhang and B.D.Qu, Phys. Rev. B 50, 698 (1994).

${ }^{14}$ W.Y. Shih, W-H Shih and I.A. Aksay Phys. Rev. B 50, 15575 (1994).

${ }^{15}$ G. Shirane, R. Pepinsky and B.C. Frazer, Acta Crystallogr., 9, 131 (1956).

${ }^{16}$ We have assumed above that the the the distortion of the unit cell takes place along the same axis all through the sample, i.e., all the unit cells have their c axis along, say, the $\mathrm{z}$ direction. This is reasonable, since there would be enormous elastic costs involved in having adjacent unit cells distended along different directions. Accordingly, the displacement can only be along the $z$ axis, but it can be either up or down.

${ }^{17}$ We calculate $d_{0}$ from the experimental polarisation data using the equation $d_{0}(T)=P(T)\left(d_{0}\left(T_{c}\right) / P\left(T_{c}\right)\right)$; At $T_{c}$, both $d_{0}$ and $P$ are known from experiments, and $P(T)$ is known over a wide range of temperatures, so we can obtain $d_{0}(T)$. The $P(T)$ data have been taken from V.G. Gavrilyachenko, R.I. Spinko, M.A. Martinenko, and E.G. Fesenko, Sov. Phys. Solid State 12, 1203 (1970).

${ }^{18}$ C. Domb, J. Chem. Phys. 25, 783 (1956); D. J. Bergman and B. I. Halperin, Phys. Rev. B13, 2145 (1976); D. C. Mattis and T. D. Schultz, Phys. Rev. 129, 175 (1963)

19 S.N. Gadekar and T.V. Ramakrishnan, J. Phys C 13, L597 (1980); Ferroelectrics 35, 249 (1981).

${ }^{20}$ F. Jona and G. Shirane, Ferroelectric Crystals (Pergamon Press 1962).

${ }^{21}$ J.P. Remeika and A.M. Glass, Mater. Res. Bull.5, 37 (1970)

${ }^{22}$ W. Cochran, Advances in Physics 9, 387 (1960)

${ }^{23}$ K. Huang, Statistical Mechanics, John Wiley, 1963.

${ }^{24}$ K. Sheshadri and R. Lahiri, unpublished. 